\title{
CLASSIFICATION PROBLEMS OF TORIC MANIFOLDS VIA TOPOLOGY
}

\author{
MIKIYA MASUDA AND DONG YOUP SUH
}

This paper is dedicated to Professor Akio Hattori on his 7rth birthday.

\begin{abstract}
We propose some problems on the classification of toric manifolds from the viewpoint of topology and survey related results.
\end{abstract}

\section{TORIC MANIFOLD AND FAN}

A toric variety $X$ of dimension $n$ is a normal complex algebraic variety with an action of an $n$-dimensional algebraic torus $\left(\mathbb{C}^{*}\right)^{n}$ having a dense orbit. Let $X^{\prime}$ be another toric variety of complex dimension $n^{\prime}$ with an action of an $n^{\prime}$-dimensional algebraic torus $\left(\mathbb{C}^{*}\right)^{n^{\prime}}$. A map from $X$ to $X^{\prime}$ is a morphism $f: X \rightarrow X^{\prime}$ together with a homomorphism $\rho:\left(\mathbb{C}^{*}\right)^{n} \rightarrow\left(\mathbb{C}^{*}\right)^{n^{\prime}}$ such that $f(t x)=\rho(t) f(x)$ for any $t \in\left(\mathbb{C}^{*}\right)^{n}$ and $x \in$ $X$. Among toric varieties, compact smooth toric varieties, which we call toric manifolds, are well studied. In this article, we propose some problems on the classification of toric manifolds from the viewpoint of topology and survey related results.

A rational convex polyhedral cone in $\mathbb{R}^{n}$ is a cone spanned by a finitely many vectors in $\mathbb{Z}^{n}$, and it is called strong if the origin is the apex. A fan in $\mathbb{R}^{n}$ is a non-empty collection $\Delta$ of rational strongly convex polyhedral cones in $\mathbb{R}^{n}$ satisfying the following conditions:

(1) Each face of a cone in $\Delta$ is a also a cone in $\Delta$.

(2) The intersection of two cones in $\Delta$ is a face of each.

A fan $\Delta$ is called complete if the union of cones in $\Delta$ covers the entire space $\mathbb{R}^{n}$, and non-singular if every cone of dimension $k$ in $\Delta$ is spanned by $k$ integral vectors which form a part of a basis of $\mathbb{Z}^{n}$. Let $\Delta^{\prime}$ be another fan in $\mathbb{R}^{n^{\prime}}$. A map from $\Delta$ to $\Delta^{\prime}$ is a linear map from $\mathbb{R}^{n}$ to $\mathbb{R}^{n^{\prime}}$ which maps $\mathbb{Z}^{n}$ into $\mathbb{Z}^{n^{\prime}}$ and a cone in $\Delta$ into a cone in $\Delta^{\prime}$.

Date: October 30, 2018.

1991 Mathematics Subject Classification. Primary 57S15, 14M25; Secondary $57 \mathrm{~S} 25$.

Key words and phrases. Toric manifolds, fans, cohomology, Pontrjagin class, quasitoric manifolds, torus manifolds.

The first author was partially supported by Grant-in-Aid for Scientific Research 4102-17540092 and the second author was partially supported by Korea Science and Engineering Foundation Grant R11-2007-035-00000-0. 
A fundamental result in the theory of toric varieties says that the category of toric varieties is equivalent to the category of fans (see [31, Theorem 1.5 and Theorem 1.13], also [30, Theorem 4.1]). A toric variety $X$ is compact if and only if the fan $\Delta_{X}$ of $X$ is complete, and smooth if and only if $\Delta_{X}$ is non-singular. Therefore, a toric variety $X$ is a toric manifold if and only if $\Delta_{X}$ is complete and non-singular.

The complex projective space $\mathbb{C} P^{n}$ with a linear action of $\left(\mathbb{C}^{*}\right)^{n}$ is a toric manifold. A product of finitely many toric manifolds is again a toric manifold with the product action, so a product of finitely many complex projective spaces is a toric manifold. Here is a bit more nontrivial example of toric manifolds.

Example 1.1. Let $B$ be a toric manifold of complex dimension $k$. Let $\gamma_{i} \rightarrow B(i=1, \ldots, \ell)$ be $\left(\mathbb{C}^{*}\right)^{k}$-equivariant line bundles over $B$. Each $\gamma_{i}$ has a $\mathbb{C}^{*}$-action defined by scalar multiplication so that the sum $\oplus_{i=1}^{\ell} \gamma_{i}$ has an action of $\left(\mathbb{C}^{*}\right)^{k+\ell}$. Let $\underline{\mathbb{C}}$ be a trivial line bundle over $B$ with fiber $\mathbb{C}$ on which the $\left(\mathbb{C}^{*}\right)^{k}$-action is trivial. Then the projectivization of $\mathbb{C} \oplus_{i=1}^{\ell} \gamma_{i}$ has an induced action of $\left(\mathbb{C}^{*}\right)^{k+\ell}$ and is again a toric manifold.

Starting with $B$ as a point and repeating the above construction, say $n$ times, we obtain a sequence of toric manifolds:

$$
B_{n} \stackrel{p_{n}}{\longrightarrow} B_{n-1} \stackrel{p_{n-1}}{\longrightarrow} \cdots \stackrel{p_{2}}{\longrightarrow} B_{1} \stackrel{p_{1}}{\longrightarrow} B_{0}=\{\text { a point }\}
$$

where the fiber of $p_{j}: B_{j} \rightarrow B_{j-1}$ for $j=1, \ldots, n$ is a complex projective space. We call the above sequence (or often the top manifold $\left.B_{n}\right)$ an $n$-stage generalized Bott tower, and especially call it an $n$-stage Bott tower when each fiber is $\mathbb{C} P^{1}$. Note that a Hirzebruch surface is a 2-stage Bott tower. The name of Bott tower was introduced and its study was initiated by Grossberg-Karshon [17]. See [8], [9] and [25] for further study on Bott towers.

\section{EQUIVARIANT COHOMOLOGY OF A TORIC MANIFOLD}

Equivariant cohomology fits very well to the study of toric manifolds, which we shall explain in this section. We refer the reader to [18], [23] and [29] for details.

We set $T=\left(\mathbb{C}^{*}\right)^{n}$ and let $X$ be a toric manifold of complex dimension $n$ with an action of $T$. Associated with the universal principal $T$-bundle $E T \rightarrow B T$, we obtain a fibration

$$
X \stackrel{\iota}{\longrightarrow} E T \times_{T} X \stackrel{\pi}{\longrightarrow} B T
$$

where $E T \times_{T} X$ is the orbit space of $E T \times X$ by the diagonal $T$-action. The equivariant cohomology of a toric manifold $X$ is the ordinary cohomology of the total space of the above fibration, that is,

$$
H_{T}^{*}(X):=H^{*}\left(E T \times_{T} X\right)
$$


Let $X_{i}(i=1, \ldots, m)$ be invariant divisors of $X$. Since $X_{i}$ and $X$ are complex manifolds, they have canonical orientations. Let $\tau_{i}$ be the image of the unit element in $H_{T}^{0}\left(X_{i}\right)$ by the equivariant Gysin homomorphism from $H_{T}^{0}\left(X_{i}\right)$ to $H_{T}^{2}(X)$ induced by the inclusion map from $X_{i}$ to $X$. We may think of $\tau_{i}$ as the Poincaré dual of the cycle $X_{i}$ in equivariant cohomology. The invariant divisors $X_{i}$ intersect transversally. Therefore, for each subset $I$ of $\{1, \ldots, m\}$ the Poincaré dual of an intersection $\cap_{i \in I} X_{i}$ is a cup product $\prod_{i \in I} \tau_{i}$, so that the product $\prod_{i \in I} \tau_{i}$ vanishes if the intersection $\cap_{i \in I} X_{i}$ is empty. It turns out that

$$
H_{T}^{*}(X)=\mathbb{Z}\left[\tau_{1}, \ldots, \tau_{m}\right] /\left(\prod_{i \in I} \tau_{i} \mid \cap_{i \in I} X_{i}=\emptyset\right) .
$$

Since the underlying simplicial complex $\Sigma_{X}$ of the fan $\Delta_{X}$ of $X$ is given by

$$
\Sigma_{X}=\left\{I \subset\{1, \ldots, m\} \mid \cap_{i \in I} X_{i} \neq \emptyset\right\},
$$

the fact (2.2) shows that $H_{T}^{*}(X)$ is the face ring (or Stanley-Reisner ring) of the simplicial complex $\Sigma_{X}$, in particular determined by $\Sigma_{X}$. Conversely, $H_{T}^{*}(X)$ as a graded ring determines the underlying simplicial complex, that is, if $H_{T}^{*}(X) \cong H_{T}^{*}(Y)$ as graded rings, then the underlying simplicial complexes $\Sigma_{X}$ and $\Sigma_{Y}$ are isomorphic (see [3] or [37, Problem 31 in p.141]).

We note that $H_{T}^{*}(X)$ is not only a graded ring but also a graded algebra over $H^{*}(B T)$ through $\pi^{*}: H^{*}(B T) \rightarrow H_{T}^{*}(X)$ where $\pi$ is the projection in (2.1). Since $H^{*}(B T)$ is a polynomial ring generated by $H^{2}(B T)$ and $H_{T}^{2}(X)$ is additively generated by $\tau_{i}$ 's, the algebra structure can be detected once we know how $\pi^{*}(u)$ is described as a linear combination of $\tau_{i}$ 's for $u \in H^{2}(B T)$. The coefficient of $\tau_{i}$ in the linear expression of $\pi^{*}(u)$ is a linear function of $u$, so that there is a unique element $v_{i} \in H_{2}(B T)$ for each $i$ such that

$$
\pi^{*}(u)=\sum_{i=1}^{m}\left\langle u, v_{i}\right\rangle \tau_{i}
$$

where $\langle$,$\rangle denotes the natural pairing between cohomology and homol-$ ogy. Therefore, the simplicial complex $\Sigma_{X}$ together with the elements $v_{i}$ 's determines the algebra structure of $H_{T}^{*}(X)$ over $H^{*}(B T)$.

Since $T=\left(\mathbb{C}^{*}\right)^{n}$, there are natural identifications

$$
\mathbb{Z}^{n}=H_{2}(B T)=\operatorname{Hom}\left(\mathbb{C}^{*}, T\right)
$$

where the last one denotes the group of homomorphism from $\mathbb{C}^{*}$ to $T$. We denote by $\lambda_{v}$ the element in $\operatorname{Hom}\left(\mathbb{C}^{*}, T\right)$ corresponding to $v \in$ $H_{2}(B T)$. It turns out that $\lambda_{v_{i}}\left(\mathbb{C}^{*}\right)$ is the $\mathbb{C}^{*}$-subgroup of $T$ which fixes the invariant divisor $X_{i}$ pointwise. For each member $I$ of $\Sigma_{X}$ we form a cone in $H_{2}(B T) \otimes_{\mathbb{Z}} \mathbb{R}=\mathbb{R}^{n}$ spanned by vectors $v_{i}$ 's for $i \in I$. The collection of those cones (and the cone consisting of only the origin) 
agrees with the fan $\Delta_{X}$ of $X$. Therefore, the data of $\Sigma_{X}$ together with the set of vectors $\left\{v_{i}\right\}$ is equivalent to the data of the fan $\Delta_{X}$.

The restriction map $\iota^{*}: H_{T}^{*}(X) \rightarrow H^{*}(X)$ is surjective and its kernel is generated by $\pi^{*}\left(H^{2}(B T)\right)$. We set $\mu_{i}=\iota^{*}\left(\tau_{i}\right) \in H^{2}(X)$. It is the (ordinary) Poincaré dual of the cycle $X_{i}$ in $X$. Then we obtain the following well-known result from (2.2), (2.3) and (2.4):

$$
H^{*}(X)=\mathbb{Z}\left[\mu_{1}, \ldots, \mu_{m}\right] / \mathcal{I}
$$

where $\mathcal{I}$ is the ideal generated by the following two types of elements:

(1) $\prod_{i \in I} \mu_{i}$ for $I \notin \Sigma_{X}$

(2) $\sum_{i=1}^{m}\left\langle u, v_{i}\right\rangle \mu_{i} \quad$ for $u \in H^{2}(B T)$,

see [16, p.106] and [31, p.134].

\section{Classification of toric MANifolds AS VARIETIES}

Before we discuss topological classification of toric manifolds, we shall recall some known results on the classification of toric manifolds as varieties. The following is fundamental.

Proposition 3.1. For toric manifolds $X$ and $Y$ of complex dimension $n$, the following are equivalent.

(V1) $X$ and $Y$ are non-equivariantly isomorphic as varieties.

(V2) $X$ and $Y$ are weakly equivariantly isomorphic as varieties, i.e., there is an isomorphism $f: X \rightarrow Y$ together with an automorphism $\rho$ of $T=\left(\mathbb{C}^{*}\right)^{n}$ such that $f(t x)=\rho(t) f(x)$ for any $t \in T$ and $x \in X$.

(V3) The fans $\Delta_{X}$ and $\Delta_{Y}$ of $X$ and $Y$ are isomorphic (or unimodularly equivalent), i.e., there is a unimodular automorphism of $\mathbb{R}^{n}$ which maps cones in $\Delta_{X}$ to cones in $\Delta_{Y}$ bijectively.

Proof. The implication from (V2) to (V1) is trivial, and the equivalence of (V2) and (V3) follows from the fundamental result in the theory of toric varieties mentioned in Section 1. So, it suffices to prove the implication from (V1) to (V2). Suppose that there is an isomorphism $f: X \rightarrow Y$. Then it induces a group isomorphism $f_{*}: \operatorname{Aut}(X) \rightarrow$ $\operatorname{Aut}(Y)$ between the automorphism groups of $X$ and $Y$. In fact, $f_{*}(g)$ for $g \in \operatorname{Aut}(X)$ is given by $f g f^{-1}$. It is known that the automorphism group of a toric manifold is a linear algebraic group with the acting torus as a maximal algebraic torus ([31, Section 3.4]) and that maximal algebraic tori in a linear algebraic group are all conjugate ([20, Corollary $\mathrm{A}$ in p.135]). Let $T_{X}$ (resp. $T_{Y}$ ) be the maximal torus of $\operatorname{Aut}(X)$ (resp. Aut $(Y)$ ) determined by the torus acting on $X$ (resp. $Y$ ). Since $f_{*}$ is an isomorphism, $f_{*}\left(T_{X}\right)$ is a maximal algebraic torus of $\operatorname{Aut}(Y)$, so that there is an element $h \in \operatorname{Aut}(Y)$ such that $f_{*}\left(T_{X}\right)=h^{-1} T_{Y} h$. Then the composition $h f: X \rightarrow Y$ induces an isomorphism $(h f)_{*}: \operatorname{Aut}(X) \rightarrow \operatorname{Aut}(Y)$ mapping $T_{X}$ to $T_{Y}$. This implies that the isomorphism $h f$ is weakly equivariant. 
Because of the equivalence between (V1) and (V3) above, the classification problem of toric manifolds as varieties reduces to the combinatorial problem of classifying fans up to isomorphism. Based on this fact, the classification of toric manifolds of dimension $n$ as varieties has been completed in several cases. For instance,

(1) $n=2$ ([31, Theorem 1.28]), or $n=3$ and the 2nd Betti number (or Picard number) is five or less ([31, Theorem 1.34]).

(2) Smooth toric Fano varieties (i.e. toric manifolds with an ample anticanonical divisor) of dimension $n \leq 4$ ([31, Proposition 2.21] for $n=2$, [31, p.90] for $n=3$, [1] and [35] for $n=4)$.

(3) 2-stage generalized Bott towers $([22])$.

See [13], [36] and their references for further classification results.

If two toric manifolds $X$ and $Y$ are (weakly equivariantly) isomorphic as varieties, then their equivariant cohomology algebras are weakly isomorphic, i.e., there is a graded ring isomorphism $\Phi: H_{T}^{*}(Y) \rightarrow H_{T}^{*}(X)$ together with an automorphism $\rho$ of $T$ such that $\Phi(u \omega)=\rho^{*}(u) \Phi(\omega)$ for any $u \in H^{*}(B T)$ and $\omega \in H_{T}^{*}(Y)$, where $\rho^{*}$ is the automorphism of $H^{*}(B T)$ induced by $\rho$. It turns out that the converse holds $([24])$, so we have the following another equivalent statement to (V1) above:

(V4) $H_{T}^{*}(X)$ and $H_{T}^{*}(Y)$ are weakly isomorphic as algebras.

\section{TOPOLOGICAL CLASSIFICATION OF TORIC MANIFOLDS}

We shall consider the topological classification of toric manifolds. If two toric manifolds are isomorphic as varieties, then they are homeomorphic, but the converse does not hold in general. Here is a wellknown simple example.

Example 4.1. For an integer $a$, we denote by $\gamma^{a}$ the $a$ fold tensor product of the canonical line bundle $\gamma$ over $\mathbb{C} P^{1}$. Let $\underline{\mathbb{C}}$ be the trivial line bundle over $\mathbb{C} P^{1}$. Then $P\left(\underline{\mathbb{C}} \oplus \gamma^{a}\right)$ is a Hirzebruch surface (i.e., 2-stage Bott tower). It is well known that $P\left(\underline{\mathbb{C}} \oplus \gamma^{a}\right)$ and $P\left(\underline{\mathbb{C}} \oplus \gamma^{b}\right)$ are isomorphic as varieties if and only if $|a|=|b|$ (see [31, Theorem 1.28 (3)]), while they are homeomorphic if and only if $a \equiv b(\bmod 2)$.

Here is a proof of the homeomorphism classification above. The "only if" part follows from the fact that $P\left(\underline{\mathbb{C}} \oplus \gamma^{a}\right)$ is spin if and only if $a$ is even. One can also check that if $H^{*}\left(P\left(\underline{\mathbb{C}} \oplus \gamma^{a}\right)\right)$ is isomorphic to $H^{*}\left(P\left(\underline{\mathbb{C}} \oplus \gamma^{b}\right)\right)$ as graded rings, then $a \equiv b(\bmod 2)$. The proof of the "if" part is as follows. Note that $P(E)$ is homeomorphic to $P(E \otimes \eta)$ for any complex vector bundle $E$ and any complex line bundle $\eta$. Suppose $a \equiv b(\bmod 2)$. Then $b-a=2 \ell$ for some $\ell \in \mathbb{Z}$ and we have homeomorphisms

$$
P\left(\underline{\mathbb{C}} \oplus \gamma^{a}\right) \cong P\left(\left(\underline{\mathbb{C}} \oplus \gamma^{a}\right) \otimes \gamma^{\ell}\right)=P\left(\gamma^{\ell} \oplus \gamma^{a+\ell}\right) .
$$


Since both $\gamma^{\ell} \oplus \gamma^{a+\ell}$ and $\underline{\mathbb{C}} \oplus \gamma^{b}$ are over $\mathbb{C} P^{1}$ and have the same first Chern class, they are isomorphic. Hence the last space above is $P\left(\underline{\mathbb{C}} \oplus \gamma^{b}\right)$, proving the "if" part.

In fact, $P\left(\underline{\mathbb{C}} \oplus \gamma^{a}\right)$ is homeomorphic to $\mathbb{C} P^{1} \times \mathbb{C} P^{1}\left(\right.$ resp. $\left.\left.\mathbb{C} P^{2} \# \overline{\mathbb{C} P^{2}}\right)\right)$ when $a$ is even (resp. odd), where $\overline{\mathbb{C} P^{2}}$ denotes $\mathbb{C} P^{2}$ with reversed orientation.

As remarked at the end of Section 3 , equivariant cohomology determines the isomorphism type of toric manifolds as varieties. This leads us to ask how much information ordinary cohomology has for toric manifolds, and the example above shows that ordinary cohomology ring distinguishes the homeomorphism types of Hirzebruch surfaces. We ask

Problem 1. Are toric manifolds $X$ and $Y$ homeomorphic if $H^{*}(X) \cong$ $H^{*}(Y)$ as graded rings (or if $X$ and $Y$ are homotopy equivalent)?

There are infinitely many closed smooth manifolds which are homotopy equivalent to $\mathbb{C} P^{n}$ but not homeomorphic to each other for $n \geq 3$ ([19], [28]). More generally, surgery theory would imply a similar result for many toric manifolds different from $\mathbb{C} P^{n}$. So, Problem 1 might be bold but we have a feeling that most of manifolds do not have large symmetry and we do not know any counterexample to Problem 1.

We shall give some evidence supporting Problem 1,

Proposition 4.2. Problem 1 has an affirmative solution for toric manifolds of complex dimension one and two.

Proof. A toric manifold of complex dimension one is $\mathbb{C} P^{1}$, so the proposition is trivial in dimension one. Simply connected real 4-dimensional closed smooth manifolds are classified up to homeomorphism by isomorphism classes of the bilinear forms defined by the intersection paring of real 2-cyclyes ([15]), so the homeomorphism types of those manifolds are distinguished by their cohomology rings. This together with the fact that any toric manifold is smooth and simply connected ([16, Section 3.2]) implies the proposition in dimension two.

Remark. Any toric manifold of complex dimension two is obtained by blowing up $\mathbb{C} P^{2}$ or a Hirzebruch surface finitely many times ([31, Theorem 1.28]). As remarked before, any Hirzebruch surface is homeomorphic to either $\mathbb{C} P^{1} \times \mathbb{C} P^{1}$ or $\mathbb{C} P^{2} \# \overline{\mathbb{C} P^{2}}$. Although $\mathbb{C} P^{1} \times \mathbb{C} P^{1}$ and $\mathbb{C} P^{2} \# \overline{\mathbb{C} P^{2}}$ are not homeomorphic, they become homeomorphic after blowing up (in other words, after taking the connected sum with $\left.\overline{\mathbb{C} P^{2}}\right)$. Thus, a toric manifold of complex dimension two is homeomorphic (even diffeomorphic) to $\mathbb{C} P^{2}, \mathbb{C} P^{1} \times \mathbb{C} P^{1}$ or the connected sum of $\mathbb{C} P^{2}$ with a finite number of copies of $\overline{\mathbb{C} P^{2}}([14])$.

Besides the proposition above, there are some partial affirmative solutions to Problem 1, For instance, 
(1) $X=\left(\mathbb{C} P^{1}\right)^{n}$ and $Y$ is an arbitrary toric manifold ([26]).

(2) $X$ is a product of complex projective spaces and $Y$ is an arbitrary generalized Bott tower (6]).

(3) $X$ and $Y$ are both 2-stage generalized Bott towers ([6]).

The reader can find more partial affirmative solutions to Problem 1 in [6].

The simplicial complex $\Sigma_{X}$ associated with a toric manifold $X$ is determined by the equivariant cohomology of $X$ as explained in Section 2. As for ordinary cohomology, the number $f_{i}$ of $i$-simplices in $\Sigma_{X}$ is determined by $H^{*}(X)$. In fact, since $H_{T}^{*}(X)$ is the face ring of the simplicial complex $\Sigma_{X}$, we have

$$
\sum_{i=0}^{\infty} \operatorname{rank} H_{T}^{2 i}(X) t^{i}=1+\sum_{i=0}^{n-1} \frac{f_{i} t^{i+1}}{(1-t)^{i+1}}
$$

(see [37, Theorem 1.4 in p.54]) while since $H_{T}^{*}(X)$ is isomorphic to $H^{*}(B T) \otimes H^{*}(X)$ as graded modules, the left hand side above is equal to

$$
\frac{1}{(1-t)^{n}} \sum_{k=0}^{n} \operatorname{rank} H^{2 k}(X) t^{k} .
$$

Equating the above two and replacing $t$ by $(s+1)^{-1}$, we see that $f_{i}$ agrees with the coefficient of $s^{n-i-1}$ in $\sum_{k=0}^{n} \operatorname{rank} H^{2 k}(X)(s+1)^{n-k}$.

Although $H^{*}(X)$ contains some information on $\Sigma_{X}$ as observed above, it is not true in general that $\Sigma_{X}$ is determined by $H^{*}(X)$ as is seen in the following example.

Example 4.3. We use the fact that a maximal simplex in $\Sigma_{X}$ corresponds to a fixed point in $X$ and blowing up $X$ at a fixed point equivariantly corresponds to applying a stellar subdivision ([13, p.70]) to the simplex corresponding to the fixed point ([16, Section 2.6]).

We start with $\mathbb{C} P^{2} \times \mathbb{C} P^{1}$ with a standard action of $\left(\mathbb{C}^{*}\right)^{3}$. The simplicial complex associated with it is the suspension of the boundary complex of a triangle. Let $Y$ be a toric manifold obtained by blowing up $\mathbb{C} P^{2} \times \mathbb{C} P^{1}$ at a fixed point equivariantly. Although there are six fixed points in $\mathbb{C} P^{2} \times \mathbb{C} P^{1}$, the simplicial complex associated with $Y$ does not depend on the fixed point chosen for blowing up. Then we blow up $Y$ at a fixed point equivariantly. In this case the simplicial complex associated with the resulting toric manifold $X$ does depend on the fixed point chosen for blowing up. In fact, we obtain three different underlying simplicial complexes shown as the first three in the second line in p.192 of [31]. However, $X$ is homeomorphic to the connected sum of $\mathbb{C} P^{2} \times \mathbb{C} P^{1}$ with two copies of $\mathbb{C} P^{3}$ (with reversed orientation) regardless of the chosen fixed point. Therefore this gives a desired example. 
On the contrary, $\Sigma_{X}$ is sometimes determined by $H^{*}(X)$. For instance, this is the case when $\Sigma_{X}$ is the boundary complex of a crosspolytope ([26]). Motivated by this, we say that the simplicial complex $\Sigma_{X}$ associated with a toric manifold $X$ is rigid if $\Sigma_{X} \cong \Sigma_{Y}$ whenever $H^{*}(X) \cong H^{*}(Y)$ as graded rings. The boundary complex $\partial \Delta^{n}$ of a simplex $\Delta^{n}$ of dimension $n$ is rigid because a toric manifold with $\partial \Delta^{n}$ as the associated simplicial complex is only $\mathbb{C} P^{n}$. Moreover, the result mentioned above asserts that a join $\partial \Delta^{0} * \cdots * \partial \Delta^{0}$, which is isomorphic to the boundary complex of a crosspolytope, is rigid. We ask

Problem 2. Which simplicial complex is rigid or not rigid? In particular, is a join $\partial \Delta^{n_{1}} * \cdots * \partial \Delta^{n_{k}}$ rigid for any value of $n_{i}$ 's and any $k$ ?

As an intermediate step to Problem 1, we may ask

Problem 3. Are toric manifolds $X$ and $Y$ homeomorphic if $H^{*}(X) \cong$ $H^{*}(Y)$ as graded rings and $\Sigma_{X}$ is rigid, or more generally, if $H^{*}(X) \cong$ $H^{*}(Y)$ as graded rings and $\Sigma_{X} \cong \Sigma_{Y}$ ?

Although Problems 1 and 3 are stated in the topological category, affirmative results known so far to those problems actually hold in the smooth category. Quite generally, we may ask

Problem 4. Are two toric manifolds diffeomorphic if they are homeomorphic?

\section{Pontruagin Class OF A TORIC MANiFOLD}

A homeomorphism between closed manifolds preserves their rational Pontrjagin classes as is well known. Since the cohomology group of a toric manifold has no torsion, any homeomorphism between toric manifolds preserves their integral Pontrjagin classes. Therefore, as a step toward Problem 1 we may ask

Problem 5. If two toric manifolds have isomorphic cohomology rings, then is there an isomorphism between their cohomology rings which preserves their Pontrjagin classes?

We have an explicit description (2.5) of $H^{*}(X)$ for a toric manifold $X$, and it is known that the Chern class of $X$ is given by $\prod_{i=1}^{m}\left(1+\mu_{i}\right)$ (see [31, Theorem 3.12 in p.131]), so the Pontrjagin class $p(X)$ of $X$ is given by

$$
p(X)=\prod_{i=1}^{m}\left(1+\mu_{i}^{2}\right) .
$$

Therefore Problem 5 is purely algebraic.

The affirmative solution to Problem[1 implies the affirmative solution to Problem 5 as remarked at the beginning of this section, but the 
results of [38] and [21] show that the converse implication also holds in complex dimension three.

Problem [1 asks whether there is a homeomorphism between toric manifolds if there is an isomorphism between their cohomology rings. More strongly we may ask

Problem 6. Is any isomorphism between cohomology rings of toric manifolds induced by a homeomorphism between the manifolds? In particular, does any isomorphism between cohomology rings of toric manifolds preserve the Pontrjagin classes of the manifolds?

Some partial affirmative solution to the latter part of Problem 6 could be found in 6].

Problem 5 or the latter part of Problem 6 reminds us of a conjecture by Petrie [33, which says that if $M$ is a closed smooth manifold homotopy equivalent to $\mathbb{C} P^{n}$ and $M$ admits a non-trivial smooth action of $S^{1}$, then any homotopy equivalence between $M$ and $\mathbb{C} P^{n}$ preserves their Pontrjagin classes, i.e., $p(M)=\left(1+x^{2}\right)^{n+1}$ for a generator $x \in H^{2}(M)$. No counterexample is known and there are some partial affirmative solutions to the conjecture. Among them, it is proved in [34] that $p(M)$ is of the above form if $M$ supports an effective smooth action of $\left(S^{1}\right)^{n}$. See [11] for related results.

\section{QUASITORIC MANIFOLDS}

The theory of toric manifolds can be developed in the topological category to some extent. The pioneering work in this direction was done by Davis-Januszkiewicz in [10]. They introduced the notion of what is now called a quasitoric manifold as a topological counterpart to a toric manifold in algebraic geometry, and showed that an analogous theory can be developed for quasitoric manifolds in the topological category. We refer the reader to a book [4] by Buchstaber-Panov for further development.

A quasitoric manifold is a closed smooth manifold $M$ of real dimension $2 n$ with a smooth action of $\left(S^{1}\right)^{n}$ such that

(1) the action is locally standard (that is, the action is locally same as a faithful real $2 n$-dimensional representation of $\left(S^{1}\right)^{n}$ in the smooth category), and

(2) the orbit space $M /\left(S^{1}\right)^{n}$ is a simple convex polytope.

The restricted action of $\left(S^{1}\right)^{n}$ on a toric manifold $X$ is locally standard and the orbit space $X /\left(S^{1}\right)^{n}$ is a manifold with corners whose faces (even $X /\left(S^{1}\right)^{n}$ itself) are all contractible and any multiple intersection of faces is connected whenever it is non-empty. When $X$ is projective, there is a moment map whose image identifies $X /\left(S^{1}\right)^{n}$ with a simple convex polytope. Therefore, a projective toric manifold provides an example of a quasitoric manifold. Even if a toric manifold is not projective, it often provides an example of a quasitoric manifold. For 
example, there are non-projective toric manifolds of complex dimension three (see [31, Section 2.3]), but any toric manifold of complex dimension three with the restricted action of $\left(S^{1}\right)^{3}$ is a quasitoric manifold, which follows from a famous theorem of Steinitz (see [39, Theorem 4.1]). However, a toric manifold may fail to be a quasitoric manifold in higher dimensions.

On the other hand, it is easy to find a quasitoric but not toric manifold. For instance, $\mathbb{C} P^{2} \# \mathbb{C} P^{2}$ is a quasitoric manifold with an appropriate action of $\left(S^{1}\right)^{2}$ but not a toric manifold because it does not allow a complex structure (even an almost complex structure). See [23, Section 5] for examples of quasitoric manifolds which are not toric but allow an almost complex structure invariant under the torus action.

Let $M$ be a quasitoric manifold of dimension $2 n$ with a simple convex polytope $P$ of dimension $n$ as the orbit space and let

$$
q: M \rightarrow P=M /\left(S^{1}\right)^{n}
$$

be the quotient map. Let $M_{i}(i=1, \ldots, m)$ be a closed smooth codimension two submanifold of $M$ fixed pointwise under some $S^{1}$-subgroup of $\left(S^{1}\right)^{n}$. We call $M_{i}$ 's characteristic submanifolds of $M$. When $M$ is a toric manifold, $M_{i}$ 's are invariant divisors. Note that $q\left(M_{i}\right)$ is a facet (i.e. codimension one face) of $P$ and the map $q$ gives a one-to-one correspondence between the characteristic submanifolds of $M$ and the facets of $P$.

The group $\operatorname{Hom}\left(S^{1},\left(S^{1}\right)^{n}\right)$ of homomorphisms from $S^{1}$ to $\left(S^{1}\right)^{n}$ can naturally be identified with $\mathbb{Z}^{n}$, and we denote by $\lambda_{v}$ the element in $\operatorname{Hom}\left(S^{1},\left(S^{1}\right)^{n}\right)$ corresponding to $v \in \mathbb{Z}^{n}$. Let $v_{i}$ be a primitive element in $\mathbb{Z}^{n}$ such that $\lambda_{v_{i}}\left(S^{1}\right)$ fixes $M_{i}$ pointwise. Note that there are two choices of $v_{i}$ and the other one is $-v_{i}$. We need an orientation data (called an omniorientation in [4]) on $M$ and $M_{i}$ to make the choice of $v_{i}$ unique. When $M$ is a toric manifold, both $M$ and $M_{i}$ are complex manifolds and have canonical orientations, so that $v_{i}$ 's are uniquely determined and $v_{i}$ agrees with the edge vector corresponding to $M_{i}$ in the fan.

Let $P_{i}(i=1, \ldots, m)$ be the facets of $P$ such that $P_{i}=q\left(M_{i}\right)$. The vectors $v_{i}$ 's are assembled to define what is called the characteristic function of $M$ :

$$
\Lambda_{M}:\left\{P_{1}, \ldots, P_{m}\right\} \rightarrow \mathbb{Z}^{n} .
$$

When $M$ is a toric manifold with $P$ as the orbit space by the restricted action of $\left(S^{1}\right)^{n}$, the simplicial complex $\Sigma_{M}$ associated with $M$ agrees with the boundary complex of the dual of $P$ and $v_{i}$ 's are the edge vectors of the fan of $M$; so the characteristic function $\Lambda_{M}$ together with (the combinatorial type of) $P$ has an equivalent data to the fan of $M$.

The map $\Lambda_{M}$ above has the property that whenever $n$ facets of $P$ meet at a vertex of $P$, their images by $\Lambda_{M}$ form a basis of $\mathbb{Z}^{n}$. A 
map from the set $\left\{P_{1}, \ldots, P_{m}\right\}$ to $\mathbb{Z}^{n}$ possessing this property is called a characteristic function on $P$. It is known that any characteristic function on $P$ can be realized as the characteristic function of some quasitoric manifold over $P$ (see [5, Section 3] $]^{1}$ ).

Let $M$ and $M^{\prime}$ be quasitoric manifolds over $P$. Then $\Lambda_{M}=\Lambda_{M^{\prime}}$ if and only if there is an equivariant homeomorphism between $M$ and $M^{\prime}$ covering the identity on $P([10])$. However an equivariant homeomorphism between them does not necessarily cover the identity on $P$. In general, it covers a self-homeomorphism of $P$ preserving the face structure of $P$. The group Aut $(P)$ of self-homeomorphisms of $P$ preserving the face structure acts on the set $\operatorname{cf}(P)$ of characteristic functions on $P$ through the natural action on the set $\left\{P_{1}, \ldots, P_{m}\right\}$. One sees that $M$ and $M^{\prime}$ are equivariantly homeomorphic if and only if $\Lambda_{M}$ and $\Lambda_{M^{\prime}}$ are in the same orbit of $\operatorname{Aut}(P)$ in $\operatorname{cf}(P)$.

Dobrinskaya 12 discusses the classification of characteristic functions over a given polytope $P$. In particular, she gives a criterion of when a quasitoric manifold over a product of simplices is a toric manifold (that is, a generalized Bott tower in this case), see also [7] and [26].

It is proved in [10] that (2.5) and (5.1) hold even for any quasitoric manifold, so one may ask the problems in Sections 4 and 5 for quasitoric manifolds. A partial affirmative solution to Problem 1 for quasitoric manifolds is given in [7] and [26].

\section{TORUS MANIFOLDS}

The family of quasitoric manifolds may not contain the family of toric manifolds entirely but an analogous theory to the toric theory can be developed for quasitoric manifolds. This implies that the theory can further be extended to a certain family of manifolds containing both toric manifolds and quasitoric manifolds.

A torus manifold $M$ introduced in [18] is a closed smooth orientable manifold of dimension $2 n$ with a smooth effective action of $\left(S^{1}\right)^{n}$ having a fixed point. Obviously both toric manifolds and quasitoric manifolds are torus manifolds. A simple example of a torus manifold which is neither toric nor quasitoric is as follows.

Example 7.1. Let $S^{2 n}$ be the $2 n$-dimensional sphere identified with the following subset of $\mathbb{C}^{n} \times \mathbb{R}$ :

$$
\left\{\left(z_{1}, \ldots, z_{n}, y\right) \in \mathbb{C}^{n} \times\left.\mathbb{R}|| z_{1}\right|^{2}+\cdots+\left|z_{n}\right|^{2}+y^{2}=1\right\},
$$

and define an action of $\left(S^{1}\right)^{n}$ on $S^{2 n}$ by

$$
\left(t_{1}, \ldots, t_{n}\right) \cdot\left(z_{1}, \ldots, z_{n}, y\right)=\left(t_{1} z_{1}, \ldots, t_{n} z_{n}, y\right)
$$

\footnotetext{
${ }^{1}$ Such a quasitoric manifold is constructed in [10, Section 1.5] but it is not obvious how to give a smooth structure on the manifold.
} 
A map

$$
\left(z_{1}, \ldots, z_{n}, y\right) \rightarrow\left(\left|z_{1}\right|, \ldots,\left|z_{n}\right|, y\right)
$$

induces a homeomorphism from the orbit space $S^{2 n} /\left(S^{1}\right)^{n}$ onto the following subset of the $n$-sphere:

$$
\left\{\left(x_{1}, \ldots, x_{n}, y\right) \in \mathbb{R}^{n+1} \mid x_{1}^{2}+\cdots+x_{n}^{2}+y^{2}=1, x_{1} \geq 0, \ldots, x_{n} \geq 0\right\} .
$$

The orbit space $S^{2 n} /\left(S^{1}\right)^{n}$ is a manifold with corners and every face of the orbit space (even the orbit space itself) is contractible. The facets are images of real codimension two submanifolds $\left\{z_{i}=0\right\}$ of $S^{2 n}$ $(i=1, \ldots, n)$ under the quotient map above and the intersection of the $n$ facets consists of two points $(0, \ldots, 0, \pm 1)$ when $n \geq 2$. Therefore $S^{2 n}$ with the above action is a torus manifold which is neither toric nor quasitoric when $n \geq 2$.

A simplicial poset $\mathcal{P}$ is a finite poset with a smallest element $\hat{0}$ such that every interval $[\hat{0}, y]$ for $y \in \mathcal{P}$, that is a subposet of $\mathcal{P}$ consisting of all elements between $\hat{0}$ and $y$, is isomorphic to the set of all subsets of a finite set, ordered by inclusion. The set of all faces of a (finite) simplicial complex with empty set added forms a simplicial poset ordered by inclusion, where the empty set is the smallest element. Such a simplicial poset is called the face poset of a simplicial complex, and two simplicial complexes are isomorphic if and only if their face posets are isomorphic. Therefore, a simplicial poset can be thought of as a generalization of a simplicial complex.

Although a simplicial poset $\mathcal{P}$ is not necessarily the face poset of a simplicial complex, it is always the face poset of a finite CW complex $\Gamma(\mathcal{P})$. In fact, to each $y \in \mathcal{P} \backslash\{\hat{0}\}$, we assign a (geometrical) simplex whose face poset is $[\hat{0}, y]$ and glue those geometrical simplices according to the order relation in $\mathcal{P}$. Then we get the $\mathrm{CW}$ complex $\Gamma(\mathcal{P})$ such that all the cells are simplices and all the attaching maps are inclusions. A finite $\mathrm{CW}$ complex like $\Gamma(\mathcal{P})$ is called a simplicial cell complex. We may say that the notion of simplicial poset is equivalent to that of simplicial cell complex.

The face poset of the orbit space $S^{2 n} /\left(S^{1}\right)^{n}$ in Example 7.1 (with reversed order by inclusion) is not the face poset of a simplicial complex when $n \geq 2$. But it is the face poset of a simplicial cell complex formed from two $(n-1)$-simplices by gluing their boundaries via the identity map. It can be thought of as the dual of the boundary of the orbit space $S^{2 n} /\left(S^{1}\right)^{n}$.

The orbit space of a toric or quasitoric manifold is topologically trivial. This is also the case for the torus manifold in Example 7.1 but not always the case for an arbitrary torus manifold as the following example shows.

Example 7.2. Take a torus manifold $M$ of dimension $2 n$ and a closed smooth manifold manifold $N$ of dimension $n$, and consider the free 
action of $\left(S^{1}\right)^{n}$ on the product $N \times\left(S^{1}\right)^{n}$ given by multiplication on the second factor. We choose a free orbit for each of $M$ and $N \times$ $\left(S^{1}\right)^{n}$, remove their invariant open tubular neighborhoods and glue the resulting manifolds along their boundaries to get a new torus manifold $M^{\prime}$. The orbit space $M^{\prime} /\left(S^{1}\right)^{n}$ is the connected sum of $M /\left(S^{1}\right)^{n}$ and $N$ at interior points. Since $N$ can be arbitrary, the orbit space of a torus manifold is not necessarily topologically trivial unlike toric or quasitoric manifolds.

For a torus manifold $M$, characteristic submanifolds $M_{i}$ 's can be defined similarly to the quasitoric case and they play the role of the invariant divisors $X_{i}$ for a toric manifold $X$. Similarly to the toric case, we get a simplicial complex $\Sigma_{M}$ and vectors $v_{i}$ 's from the characteristic submanifolds $M_{i}$ 's. Using these data, one can associate with $M$ a combinatorial object $\Delta_{M}$ called the multi-fan of $M$ in a similar fashion to the toric case ([18], 23]). Precisely speaking, we assign orientations on $M$ and $M_{i}$ 's (i.e. an omniorientation) which make the choice of $v_{i}$ 's unique as remarked in Section [6 and moreover we attach an integer to each cone of maximum dimension $n$. Such a cone corresponds to $n$ characteristic submanifolds in $M$ and the integer attached to the cone counts the number of points (with sign determined by the omniorientation) in the intersection of the $n$ characteristic submanifolds. When $M$ is a toric manifold, the attached integers are all one (so that we may neglect them) and the multi-fan $\Delta_{M}$ is an ordinary fan, but unless $M$ is a toric manifold, the attached integers are not necessarily one and cones in $\Delta_{M}$ may overlap. Although $\Delta_{M}$ contains a lot of geometrical information on $M$, it does not determine $M$ in general. For instance, the torus manifolds $M$ and $M^{\prime}$ in Example 7.2 are not equivariantly homeomorphic in general, but their mutli-fans are same because a mulit-fan is defined using only characteristic submanifolds and the characteristic submanifolds of $M$ and $M^{\prime}$ are same.

If the action on a torus manifold of dimension $2 n$ is locally standard, then its orbit space is a compact nice manifold of dimension $n$ with corners, where "nice" means that there are exactly $n$ codimensionone faces meeting at each vertex. A teardrop (of dimension two) is a manifold with corners but not nice. In order to define a good family of torus manifolds, we shall introduce some terminology for a compact nice manifold with corners.

Let $Q$ be a compact nice manifold with corners. Faces of $Q$ can naturally be defined and we understand that $Q$ itself is a face. We say that $Q$ is a homology cell if all faces of $Q$ are acyclic, and a homology polytope if it is a homology cell and any multiple intersection of faces is connected whenever it is non-empty. We also say that $Q$ is a homotopy cell (resp. homotopy polytope) if it is a homology cell (resp. homology polytope) and all faces are simply connected so that all faces are contractible. A simple convex polytope is a homotopy polytope and the 
orbit space $S^{2 n} /\left(S^{1}\right)^{n}$ in Example 7.1 is a homotopy cell but not a homotopy polytope when $n \geq 2$. The face poset of $Q$ (with reversed order by inclusion) is a simplicial poset and is the face poset of a simplicial complex if any multiple intersection of faces of $Q$ is connected whenever it is non-empty. The following question asks whether homotopy cells or homotopy polytopes can be determined combinatorially.

Problem 7. Are homotopy cells (or homotopy polytopes) homeomorphic as manifold with corners if their face posets are isomorphic?

It is shown in 25] that if $H^{\text {odd }}(M)=0$ for a torus manifold $M$, then the torus action on $M$ is locally standard, and moreover shown that the orbit space of a locally standard torus manifold $M$ is a homology cell (resp. homology polytope) if and only if $H^{o d d}(M)=0\left(\operatorname{resp} . H^{*}(M)\right.$ is generated by $H^{2}(M)$ as a ring). However, the orbit space itself or its faces may have a non-trivial fundamental group. Since a torus group is connected, simply connectedness of a space with torus action is inherited to its orbit space ([2, Corollary 6.3 in p.91]). Therefore, if a torus manifold $M$ satisfies the following two conditions:

(1) $H^{\text {odd }}(M)=0$ (resp. $H^{*}(X)$ is generated by $H^{2}(M)$ as ring),

(2) $M, M_{i}$ 's and connected components of any multiple intersection of $M_{i}$ 's are all simply connected,

then the orbits space $M /\left(S^{1}\right)^{n}$ is a homotopy cell (resp. homotopy polytope). We believe that torus manifolds satisfying the two conditions above will constitute a good family of manifolds for which the toric theory can be developed in the topological category in a nice way. Toric or quasitoric manifolds are contained in this family.

As pointed out in Section 2, the data of the fan of a toric manifold $X$ is equivalent to the data of the simplicial complex $\Sigma_{X}$ together with the set of vectors $\left\{v_{i}\right\}$. For a torus manifold $M$, we still have the vectors $\left\{v_{i}\right\}$ and the face poset of $M /\left(S^{1}\right)^{n}$ takes the place of the simplicial complex $\Sigma_{X}$. The same argument as in [10] will show that the homeomorphism type of a torus manifold $M$ satisfying the two conditions above will be determined by the face poset of $M /\left(S^{1}\right)^{n}$ together with the set of vectors $\left\{v_{i}\right\}$ if Problem 7 is affirmatively answered. We may ask the problems in Sections 4 and 5 even for those torus manifolds. The reader can find related study in dimension 4 ([32]) and in dimension 6 ([27]).

\section{REFERENCES}

[1] V. V. Batyrev, On the classification of toric Fano 4-folds, Algebraic geometry, 9. J. Math. Sci. (New York) 94 (1999), 1021-1050.

[2] G. E. Bredon, Introduction to Compact Transformation Groups, Academic Press, New York-London, 1972.

[3] W. Bruns and J. Gubeladze, Combinatorial invariance of Stanley-Reisner rings, Georgian Math. J. 3 (1996), 315-318. 
[4] V. M. Buchstaber and T. E. Panov, Torus Actions and Their Applications in Topology and Combinatorics, University Lecture, vol. 24, Amer. Math. Soc., Providence, R.I., 2002.

[5] V. M. Buchstaber, T. E. Panov and N. Ray, Spaces of polytopes and cobordism of quasitoric manifolds, arXiv:math.AT/0609346.

[6] S. Choi, M. Masuda and D. Y. Suh, Topological classification of generalized Bott towers, in preparation.

[7] S. Choi, M. Masuda and D. Y. Suh, Quasitoric manifolds over a product of simplices, in preparation.

[8] Y. Civan, Bott towers, crosspolytopes and torus actions, Geom. Dedicata 113 (2005), 55-74.

[9] Y. Civan and N. Ray. Homotopy decompositions and K-theory of Bott towers, $K$-Theory 34 (2005), 1-33.

[10] M. W. Davis and T. Januszkiewicz, Convex polytopes, Coxeter orbifolds and torus actions, Duke Math. J. 62 (1991), 417-451.

[11] A. Dessai, Torus actions on homotopy complex projective spaces, Math. Z. 247 (2004), 505-511.

[12] N. E. Dobrinskaya, Classification problem for quasitoric manifolds over a given simple polytope, Functional Analysis and its Applications 55 (2001), 83-89.

[13] G. Ewald, Combinatorial Convexity and Algebraic Geometry, Graduate Texts in Mathematics, vol. 168, Springer-Verlag, New York, 1996.

[14] S. Fischl and D. Yavin, Which 4-manifolds are toric varieties?, Math. Z. 215 (1994), 179-185.

[15] M. Freedman, The topology of four-dimensional manifolds, J. Differential Geom. 17 (1982), 357-453.

[16] W. Fulton, An Introduction to Toric Varieties, Ann. of Math. Studies, vol. 113, Princeton Univ. Press, Princeton, N. J., 1993.

[17] M. Grossberg and Y. Karshon, Bott towers, complete integrability, and the extended character of representations. Duke Math. J. 76 (1994), 23-58.

[18] A. Hattori and M. Masuda, Theory of multi-fans, Osaka J. Math. 40 (2003), $1-68$.

[19] W. C. Hsiang, A note on free differentiable actions of $S^{1}$ and $S^{3}$ on homotopy spheres, Ann. of Math. 83 (1966), 266-272.

[20] J. E. Humphreys, Linear Algebraic Groups, Graduate Texts in Mathematics, vol. 21, Springer-Verlag, New York, 1987.

[21] P. E. Jupp, Classification of certain 6-manifolds, Math. Proc. Camb. Phil. Soc. 73 (1973), 293-300.

[22] P. Kleinschmidt, A classification of toric varieties with few generators, Aequationes Mathematicae 35 (1988), 254-266.

[23] M. Masuda, Unitary toric manifolds, multi-fans and equivariant index, Tohoku Math. J. 51 (1999), 237-265.

[24] M. Masuda, Equivariant cohomology distinguishes toric manifolds, arXiv:math.AT/0703330.

[25] M. Masuda and T. Panov, On the cohomology of torus manifolds, Osaka J. Math. 43 (2006), 711-746.

[26] M. Masuda and T. Panov, Semifree circle actions, Bott towers, and quasitoric manifolds, arXiv:math.AT/0607094.

[27] D. McGavran, $T^{3}$-actions on simply connected 6-manifolds, I, Trans. Amer. Math. Soc. 220 (1976), 59-85.

[28] D. Montgomery and C. T. Yang, Differentiable actions on homotopy seven spheres, Trans. Amer. Math. Soc. 122 (1966), 480-498. 
[29] G. Mukherjee (ed.), Transformation Groups, Symplectic Torus Actions and Toric Manifolds, with contributions by C. Allday, M. Masuda and P. Sankaran, Hindustan Book Agency, India 2005.

[30] T. Oda, Lectures on Torus embeddings and Applications (Based on Joint Work with Katsuya Miyake), Tata Inst. of Fund. Research 58, Springer, Berlin, Heidelberg, New York, 1978.

[31] T. Oda, Convex Bodies and Algebraic Geometry. An Introduction to the Theory of Toric Varieties, Ergeb. Math. Grenzgeb. (3), 15, Springer-Verlag, Berlin, 1988.

[32] P. Orlik and F. Raymond, Actions of the torus on 4-manifolds, I, Trans. Amer. Math. Soc. 152 (1970), 531-559.

[33] T. Petrie, Smooth $S^{1}$-actions on homotopy complex projective spaces and related topics, Bull. Amer. Math. Soc. 78 (1972), 105-153.

[34] T. Petrie, Torus actions on homotopy complex projective spaces, Invent. Math. 20 (1973), 139-146.

[35] H. Sato, Toward the classification of higher-dimensional toric Fano varieties, Tohoku Math. J. 52 (2000), 383-413.

[36] H. Sato, Smooth toric Fano five-folds of index two, Proc. Japan Acad. Ser. A Math. Sci. 82 (2006), 106-110.

[37] R. P. Stanley, Combinatorics and Commutative Algebra, second edition, Progress in Math. 41, Birkhäuser, Boston, 1996.

[38] C. T. C. Wall, Classification problem in differential topology. V : On certain 6-manifolds, Invent. Math. 1 (1966), 355-374.

[39] G. M. Ziegler, Lectures on polytopes, Graduate Texts in Mathematics, vol. 152, Springer-Verlag, New York, 1995.

Department of Mathematics, Osaka City University, Sugimoto, SumiyoshiKU, OSAKA 558-8585, JAPAN

E-mail address: masuda@sci.osaka-cu.ac.jp

Department of Mathematics, Korea Advanced Institute of Science And Technology, Gu-Sung Dong, Yu-sung Gu, Daejeon 305-701, Korea

E-mail address: dysuh@math.kaist.ac.kr 